\title{
Fault Identification, Classification, and Location on Transmission Lines Using Combined Machine Learning Methods
}

\author{
Nguyen Nhan Bon ${ }^{1}$, Le Van Dai ${ }^{2}{ }^{*}$ \\ ${ }^{1}$ Faculty of Electrical and Electronics Engineering, Ho Chi Minh University of Technology an Education, \\ Ho Chi Minh City, Vietnam \\ ${ }^{2}$ Faculty of Electrical Engineering Technology, Industrial University of Ho Chi Minh City, Ho Chi Minh City, Vietnam \\ Received 29 April 2021; received in revised form 02 June 2021; accepted 03 June 2021
}

DOI: https://doi.org/10.46604/ijeti.2022.7571

\begin{abstract}
This study develops a hybrid method to identify, classify, and locate electrical faults on transmission lines based on Machine Learning (ML) methods. Firstly, Wavelet Transform (WT) technique is applied to extract features from the current or voltage signals. The extracted signals are decomposed into eleven coefficients. These coefficients are calculated to the energy level, and the data of teen fault types are converted to the RGB image. Secondly, GoogLeNet model is applied to classify the fault, and Convolutional Neural Network (CNN) method is proposed to locate the fault. The proposed method is tested on the four-bus power system with the $220 \mathrm{kV}$ transmission line via time-domain simulation using Matlab software. The conditions of the fault resistance random values and the pre-fault load changes are considered. The simulation results show that the proposed method has high accuracy and fast processing time, and is a useful tool for analyzing the system stability in the field of electricity.
\end{abstract}

Keywords: machine learning, fault identification, fault classification, fault location

\section{Introduction}

The electric system is a collection of many different components including the complex dynamic and interactional elements, which can always be leading to disturbance or fault, especially electrical faults. Electrical faults are caused by weather conditions, miss operation, overload, equipment failures, human errors, and smoke of fires. In the past decades, power systems have faced various severe blackouts due to technical, human, and electrical faults [1-2]. The effects caused by them are the overcurrent flow, danger to person, equipment loss, economic loss, and national security.

The faults in a three-phase system can be classified into symmetrical and unsymmetrical faults. For the symmetrical faults, they can also be called the balanced faults and are of two types, namely Three Line-to-Ground (LLLG) and Three Line (LLL) faults. In this case, it is a very serious fault and occurs in frequency. For the unsymmetrical faults, they can also be called unbalanced faults and are of three types, namely Single Line-to-Ground (SLG), Line-to-Line (LL), and Double Line-to-Ground (LLG). In this case, it is very popular and less serious than the symmetrical faults. Table 1 shows the occurred probability of different types of faults, and the possibility of fault on various parts of the electric system is listed in Table 2 [3]. Whenever a fault occurs on any part of the electric system, different degrees of consequences will be generated for the entire system. Observing from Table 2, 50\% of fault can occur for the transmission line. To solve this problem, fault identification, classification, and location on the transmission line have been studied for the past decade. It is also the motivation for the authors to propose this study.

* Corresponding author. E-mail address: levandai@iuh.edu.vn

Tel.: +84-0984.160178; Fax: +84-02839940954 
Table 1 Occurrence probability of different types of faults

\begin{tabular}{|c|c|}
\hline Types of fault & Occurrence probability (\%) \\
\hline Single Line-to-Ground (SLG) & 85 \\
\hline Double Line-to-Ground (LLG) & 5 \\
\hline Line-to-Line (LL) & 8 \\
\hline Three Line (LLL) and Three Line-to-Ground (LLLG) & 2 \\
\hline
\end{tabular}

Table 2 Possibility of fault on various parts of the electric system

\begin{tabular}{|c|c|}
\hline Types of equipment & Occurred fault (\%) \\
\hline Transmission line & 50 \\
\hline Switchgear & 15 \\
\hline Transformer & 12 \\
\hline Cables & 10 \\
\hline Miscellaneous & 8 \\
\hline Control equipment & 3 \\
\hline Current Transformer (CT) and Potential Transformer (PT) & 2 \\
\hline
\end{tabular}

This study proposes a hybrid approach using Wavelet Transform (WT), GoogLeNet, and Convolutional Neural Network (CNN) to identify, classify, and locate other fault types for transmission lines. WT is divided into Discrete WT (DWT) and Continuous WT (CWT). For the analysis of the transients in the power system, DWT is used more than CWT because it can easily obtain the characteristics of the voltage and current signals of the multiple frequency bands. Seven signals including the currents and voltages of phases $A, B, C$, and neutral current are considered. After calculating seven signals including current and voltage ones, it obtains a state vector having 28 elements, and this vector is considered the operating status of the power system. The wavelet features are converted to RGB image format, having the size of $224 \times 224 \times 3$ that is considered the input of GoogLeNet. GoogLeNet is used to train and test RGB images $(224 \times 224 \times 3)$ with the purpose to achieve the classification of different fault states. $\mathrm{CNN}$ is applied to identify and locate the fault location.

The novelty and contribution of this study include the following aspects: (i) Introducing a hybrid approach by associating WT, GoogLeNet, and CNN is a productive tool to identify, classify, and locate the symmetrical and unsymmetrical faults on the transmission lines. (ii) GoogLeNet is proposed to classify the fault in the transmission line with input to be RGB images. (iii) By using the proposed method, the tested and training results are highly accurate with fast processing time and simple implementation.

This study is organized for the remanent parts as follows: Literature review is provided in section 2. Section 3 presents the methodology of the WT technique, GoogLeNet, and CNN technique, and then develops the hybrid method to identify, classify, and locate the symmetrical and unsymmetrical faults on the transmission lines. Section 4 implements and evaluates the proposed method based on the four-bus power system using Matlab software via the simulation cases. The result is also presented in this section. Finally, the conclusion is outlined in section 5.

\section{Literature Review}

Improving the power quality and ensuring the system protection, the fault identification, classification, and location on the transmission line are the first stage and also are the biggest challenge to each research scientist, engineer, and distribution company when designing, operating, and protecting the power system. For this purpose, in the last decades, some Machine Learning (ML) methods have been introduced as Support Vector Machine (SVM), Artificial Neural Network (ANN), Fuzzy Logic Control (FLC), and other methods. For SVM, it is an intelligent classification technique and it was originally introduced by Vapmik et al. [4]. This method uses the input vector to map into the feature space having an effective and efficient tool for 
detecting and classifying faults in the power transmission line [5]. There is a lot of research developed based on SVM that are tested and implemented practically. Ray et al. [6] have applied SVM for the fault classification and location of a long transmission line. Many combinative methods with SVM are used to identify and classify the faults on the transmission line. Babu et al. [7] use the SVM and Empirical Mode Decomposition (EMD) methods, Guo et al. [8] apply SVM and Principal Component Analysis (PCA) methods, Malathi et al. [9] use the multi-class SVM approach, and Livani et al. [10] use a method of combining DWT with SVM. All these techniques are based on continuous monitoring of current, voltage, and impedance.

ANN is a programming technique developed in different categories of structures. This method is simple in implementation and very powerful for identifying and classifying faults [11]. There is a lot of research developed based on ANN [12]. ANN is discussed in [13-15] using WT to obtain the peculiar features as energy from the obtained signals of current and voltage that are further applied in ANN for identifying and classifying faults. The obtained simulation results are highly accurate. However, its disadvantage is the large training time. FLC is also a very powerful method for identifying and classifying faults and is applied in [16-18]. Saradarzadeh et al. [16] have proposed a fuzzy structure to identify the fault from the separate voltage and current signals using the phase sequence ingredients of three-phase voltage and current that can be measured from the protection relays. Prasad et al. [17] used a fuzzy structure by two classifiers. The first classifier is applied for identifying ground faults and the other classifier is applied for detecting phase faults. Adhikari et al. [18] applied fuzzy logic as input for field-programmable gate array from the obtained three-phase current from Compact Reconfigurable Input/Output (CRIO) devices.

SVM, ANN, or FLC can be applied to other problems, each with their typical application concentrates depending on different complexity or nature of the issue to be solved. However, each method has its disadvantage. In the case of ANN, there is no exact rule for the selection of the number of hidden layers and neurons for each hidden layer. SVM requires a huge matrix storge [19]. FLC is difficult to model mathematically and requires expert knowledge [20]. In addition, there are other techniques. Farshad et al. [21] used k-Nearest Neighbors (k-NN) to classify and locate SLG faults. Ray [22] applied extreme learning machine to the fault location in series compensated transmission line. Hosseini [23] introduced a combination of WT and SVM. Haq et al. [24] introduced a combination of ML based on DWT and double channel extreme ML.

More recently, some accurate hybrid methods have been introduced to identify, classify, and locate the fault in the power system. For example, Ray et al. [25] have presented a method combining learning machine and s-transform to locate the fault for underground power in the distribution system. The use of ANN and WT has been introduced in [26] to study fault detection and classification in the long transmission line. The method based on WT and fuzzy logic has been proposed in [27] to the eleven fault classification in the transmission line. In this method, WT has been applied to decompose the output signals, and fuzzy logic has been applied to the rules to give fault types as output. Another fault location technique has been developed by Jung et al. [28] for transmission systems using WT and neuro-fuzzy. For this technique, wavelet Multi-Resolution Analysis (MRA) coefficients are used for detecting and classifying faults.

As known, the WT technique is extensively developed in the 1980s and used in the applied mathematics field for image and signal processing. WT became a tool used for analyzing electromagnetic transients. Since then, WT is widely applied as a feature extraction method in other fault diagnosis systems based on the characteristics of voltage and current signals in frequency bands. CNN is a feed-forward neural network which is considered one of the most excellent techniques in the image classification field, and its structure assures a sure extent of scale and translation. GoogLeNet is an efficient CNN presented in ImageNet Large Scale Visual Recognition Challenge 2014 (ILSVRC14), which is developed by researchers at Google. In recent years, many techniques such as WT [29-32], GoogLeNet [33], and CNN [34] have been explored for the control and stability in power systems including fault identification, classification, and location. 


\section{The Methodology and Proposed Method}

\subsection{The wavelet transform (WT) technique}

The WT technique is extensively developed in the 1980s and used in the applied mathematics field for image and signal processing. WT became a tool used for analyzing electromagnetic transients. Since then, WT is widely applied as a feature extraction method in other fault diagnosis systems based on the characteristics of voltage and current signals in frequency bands. WT is divided into two types namely DWT and CWT. For the analysis of the transients in the power system, DWT is used more than CWT because it can easily obtain the characteristics of the voltage and current signals of the multiple frequency bands. It notes that when implementing DWT, the choice of mother wavelet and dissociation level has been included before creating the features.

The continuous mother wavelet is defined as follows [31]:

$$
w_{s, \tau}(t)=\frac{1}{\sqrt{s}} w\left(\frac{t-\tau}{s}\right) ; \text { with } s, \tau \in R, \text { and } s \neq 0
$$

where $s$ is the scale parameter relating to the location of the window and it is defined as the time information in the transformed domain; $\tau$ is the translation parameter relating to the information in the frequency of the signal and it is defined as the width of the window.

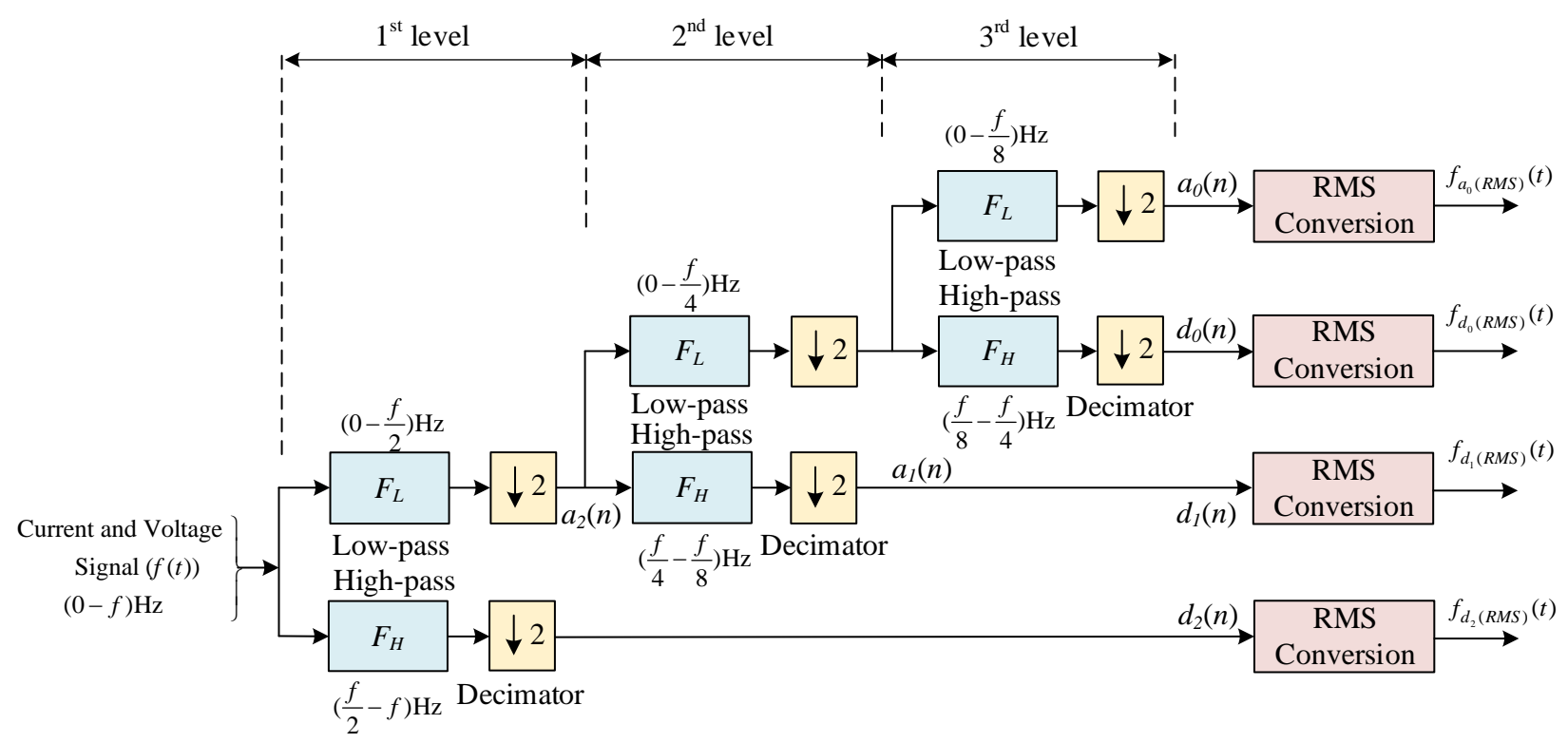

Fig. 1 Process of DWT decomposition

DWT in combination with MRA is used to analyze the discreteness based on the discrete values of the scales $m$ and translation $n$. The DWT-MRA with a signal $f(t)$ is calculated by passing a series of filters that are the low-pass and high-pass ones. In this study, DWT is applied to extract features from the current or voltage signals on the transmission line as illustrated in Fig. 1. Observing this figure, at each level, it encloses a set of approximate $(a)$ and detail $(d)$ factors. These factors are respectively calculated as follows [35]:

$$
\begin{aligned}
& a_{m}(n)=\int_{-\infty}^{\infty} f(t) \varphi_{m, n}(t) d t \\
& d_{m}(n)=\int_{-\infty}^{\infty} f(t) w_{m, n}(t) d t
\end{aligned}
$$


where $\varphi_{m, n}(t)$ and $w_{m, n}(t)$ are the scaling and wavelet functions, and are respectively calculated based on the choice of the mother wavelet as follows:

$$
\begin{gathered}
\varphi_{m, n}(t)=\frac{1}{\sqrt{s_{0}^{m}}} \varphi\left(\tau_{0}^{m} t-n\right) ; \text { with } m, n \in Z \\
w_{m, n}(t)=\frac{1}{\sqrt{s_{0}^{m}}} w\left(\tau_{0}^{m} t-n\right) ; \text { with } m, n \in Z
\end{gathered}
$$

The corresponding relationship of the scaling and wavelet functions between two levels $m$ and $m+1$ is calculated by applying Mallet algorithm [36]. The low-pass and high-pass filters ( $F_{L}$ and $F_{H}$, respectively) can be determined by using Matlab software:

$$
\begin{aligned}
& c_{m}(n)=\sum_{\zeta} F_{L}(\zeta-2 n) c_{m+1}(\zeta) \\
& d_{m}(n)=\sum_{\zeta} F_{H}(\zeta-2 n) c_{m+1}(\zeta)
\end{aligned}
$$

The main purpose of classifying and identifying the fault is to find the change between normal and fault states based on the received signal. The change level can be calculated through the Root Mean Square (RMS) values of the $a$ and $d$ factors in each wavelet level, and be regarded as the energy value of the considered wavelet level. The scaling and wavelet factors are respectively determined as follows:

$$
\begin{aligned}
& f_{a_{0}(R M S)}(t)=\sqrt{\frac{\sum_{n}\left[a_{0}(n)\right]^{2}}{2^{3} L_{a_{0}}}} ; \text { with } m=3 \div 1 \\
& f_{d_{m}(R M S)}(t)=\sqrt{\frac{\sum_{n}\left[d_{m}(n)\right]^{2}}{2^{3-m} L_{d_{3-m}}}} ; \text { with } m=3 \div 1
\end{aligned}
$$

where $L_{a_{0}}$ is the number of points in the scaling factor, $L_{d_{3-m}}$ is the number of points in wavelet factor $d_{3-m}$, and the parameters $m$ and $n$ are the multi-resolution levels and translation, respectively.

In this study, the $s_{0}$ value is chosen to be 2 , the $\tau_{0}$ value is chosen to be 2 , and the Daubechies (db4) is applied for decomposition to the level of 3 (the total resolution level is to be 3 ). Therefore, the wavelet factors are used to calculate to be $a_{0}, d_{0}, d_{1}$, and $d_{2}$.

The RMS signal vector can be calculated based on the scaling and wavelet factors at the three resolution levels as follows:

$$
\mathrm{RMS}_{\text {Signal }}=\left[\begin{array}{l}
\left\|f_{a_{0}(R M S)}(t)\right\|,\left\|f_{d_{0}(R M S)}(t)\right\| \\
\left\|f_{d_{1}(R M S)}(t)\right\|,\left\|f_{d_{2}(R M S)}(t)\right\|
\end{array}\right]
$$

For this study, seven signals including the currents and voltages of phases $A, B, C$, and neutral current are considered. As assumed above, each signal is dissociated by four WT's factors. Therefore, each vector is considered to examine 28 elements. In general, each signal is dissociated into a feature vector including four elements as follows: 


$$
\mathbf{u}_{x}=\left[\begin{array}{l}
\left\|U_{x(R S M S) a_{0}}\right\|,\left\|U_{x(R S M S) d_{0}}\right\| \\
\left\|U_{x(R S M S) d_{1}}\right\|,\left\|U_{x(R S M S) d_{2}}\right\|
\end{array}\right]^{\mathrm{T}}
$$

After calculating seven signals including current and voltage ones, it obtains a state vector having 28 elements, and this vector is considered the operating status of the power system.

\subsection{GoogLeNet}

GoogLeNet is an efficient CNN presented in ILSVRC14, which is developed by researchers at Google. Its architecture is introduced in [37]. The overall network architecture is shown in Fig. 2. In general, it has 22 layers. In order to increase the number of units in each layer, the parallel filter with the sizes of $1 \times 1,3 \times 3$, and $5 \times 5$ is called the inception module. In this architecture, there are nine inception modules as shown in Fig. 2, and their architecture is shown in the zoom-in called dimensionality reduction form. The use of this form allows for considerable increases in the units at each stage without having a sharp increase in necessary computational resources later.

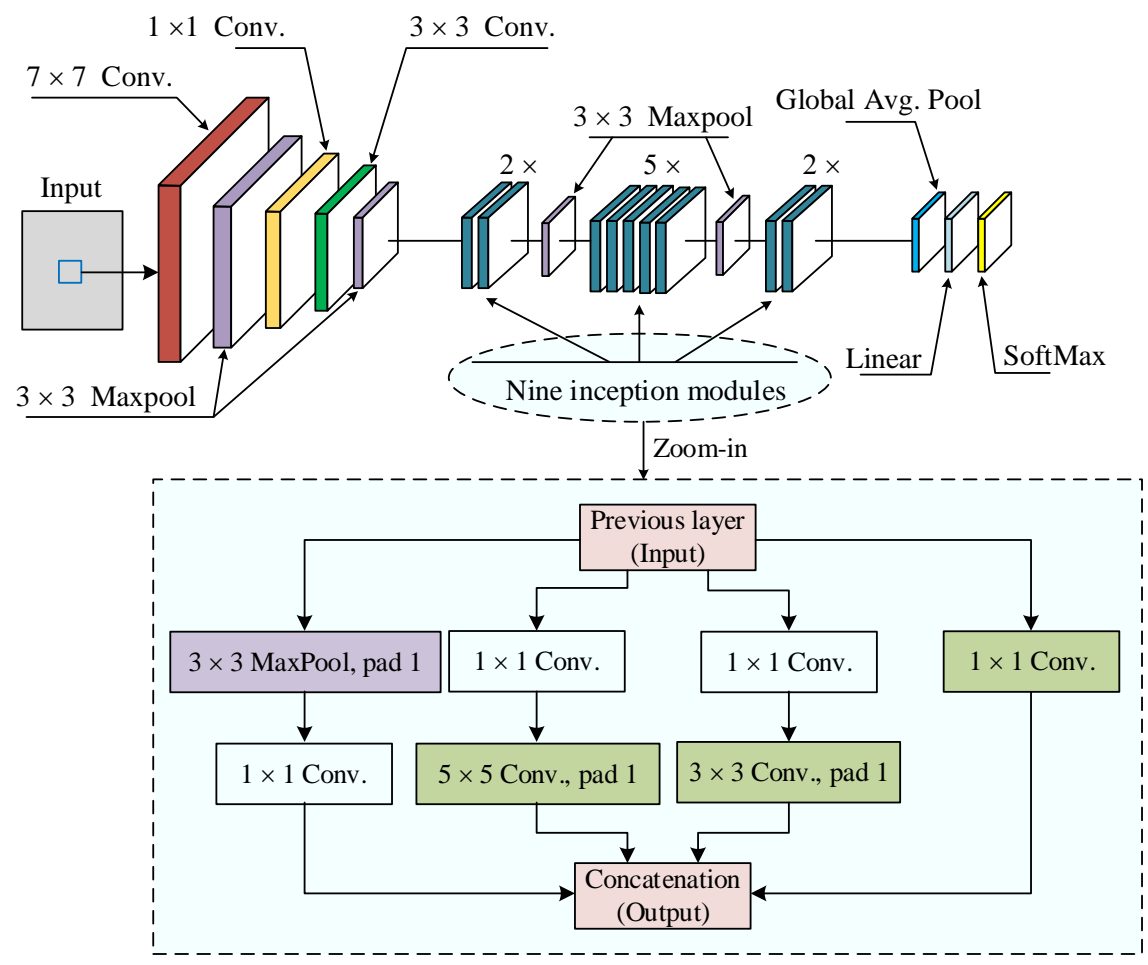

Fig. 2 The GoogLeNet structure

Despite the fact that GoogLeNet architecture has 22 layers of deep CNN, the number of parameters is 12 times less than AlexNet and its accuracy is significantly better. All the convolution, reduction, and projection layers apply the Rectified Linear Unit (ReLU) non-linearity. The reduction and projection layers have used the average pooling layer to replace the fully connected layers, whereas the first few inception modules have used auxiliary classifiers. For this purpose, it can combat the vanishing gradient problem and overfitting problem. In this study, the input of GoogLeNet is the RGB image having the size of $224 \times 224 \times 3$.

\subsection{The convolutional neural network $(C N N)$ technique}

CNN is considered one of the most excellent techniques in the image classification field, and its structure assures a sure extent of scale and translation. A five-layer CNN structure is shown in Fig. 3, in which it has two convolutional layers notating $\mathrm{C} 1$ and $\mathrm{C} 3$, two subsampling layers notating S2 and S4, and a fully connected layer notating V5. 


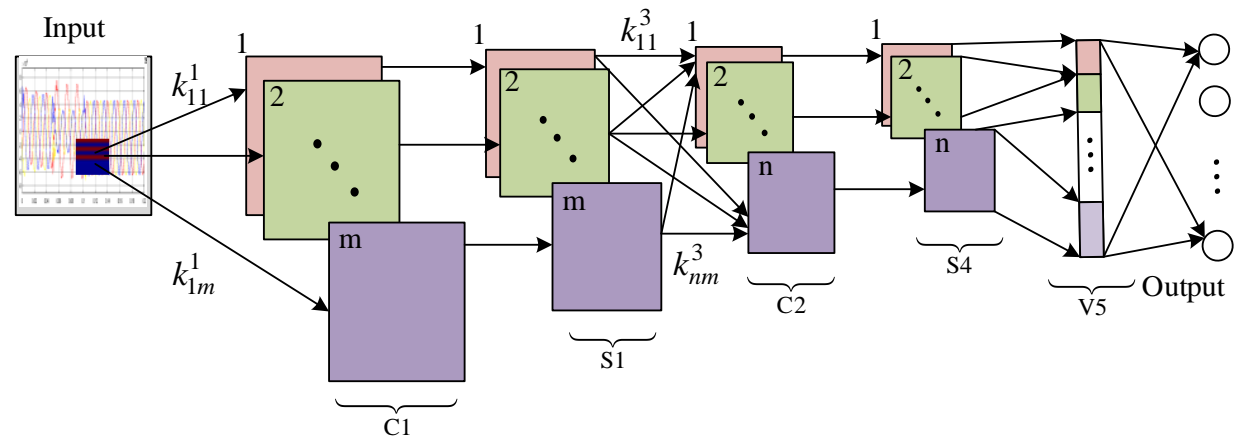

Fig. 3 The CNN structure

Convolution layers (C1 and C3): The convolution layer operates as a specific linear process. It is used to extract feature maps from the image of the previous layer, and $\mathrm{C} 1$ is especially used to extract the local features of input data.

Assuming that a pixel image $P$ has a size of $A_{p} \times B_{p}$ and can be described by a function of the grey level of the pixel in $x_{p} t h$ row and $y_{p}$ th column and the convolution kernel with a size of $k_{a} \times k_{b}$, the convolution of image $P$ and kernel can be calculated as follows [38]:

$$
C\left(s_{c}, t_{c}\right)=g\left(x_{p}, y_{p}\right) \times k\left(x_{p}, y_{p}\right)=\sum_{x_{p}=1}^{k_{a}} \sum_{y_{p}=1}^{k_{b}} k\left(x_{p}, y_{p}\right) g\left(s+x_{p}-1, t+y_{p}-1\right)
$$

where $C\left(\right.$ ) is the convolution result, $g()$ is the function of the grey level, $k()$ is the convolution kernel, and $s_{c}$ and $t_{c}$ satisfy the following condition:

$$
\left\{\begin{array}{l}
1 \leq s_{c} \leq A_{p}-k_{a}+1 \\
1 \leq t_{c} \leq B_{p}-k_{b}+1
\end{array}\right.
$$

The feature maps of the current convolutional layer can be obtained after adding bias, and can be expressed by the following activation function:

$$
x_{c_{j}}^{l}=f\left(\sum_{i \in M_{c_{j}}} x_{i}^{l-1} k_{i c_{j}}^{l}+b_{c_{j}}^{l}\right)
$$

where $l$ is the number of the network layer; $k_{i c_{j}}^{l}$ and $b_{c_{j}}^{l}$ are the convolution kernel and bias corresponding to the feature map $c_{j}$ in the $l$-layer, respectively; $M_{c}$ is the input set of feature images; $x_{c_{j}}^{l}$ and $x_{i}^{l-1}$ are the $l$-layer output and input, respectively; $f($ ) is the activation function.

Subsampling layers (S2 and S4): The subsampling layer is used to scale and map the data of the previous layer, which can reduce the data dimension and calculation complexity and can prevent over-befitting. If the input matrix has the size of $n_{s} \times n_{s}$, the pooling matrix has the size of $k_{s} \times k_{s}$, and the pooled output matrix has the size of $m_{s} \times m_{s}$. The feature maps of the current subsampling layer can be obtained as follows:

$$
x_{s_{j}}^{l}=f\left(\frac{1}{k_{s}} \sum_{i \in M_{s}} x_{i}^{l-1} k_{i s_{j}}^{l}+b_{s_{j}}^{l}\right)
$$

where $k_{i s_{j}}^{l}$ and $b_{s_{j}}^{l}$ are the convolution kernel and bias corresponding to the feature map $s_{j}$ in the $l$-layer, respectively; $x_{s_{j}}^{l}$ and $x_{i}^{l-1}$ are the $l$-layer output and input, respectively; $n_{s}, m_{s}$, and $k_{s}$ satisfy the following relationship: 


$$
k_{s}=\frac{n_{s}}{m_{s}}
$$

Fully connected layer (V5) and output layer: This layer is typically used in the top layer of CNN for the purpose of capturing the complex relationships between high-level features. All the feature maps after convolution and sampling are mixed into column vectors one by one, which are fully connected to the output layer. In this study, each unit in the output layer is considered to calculate the dot product of the feature vector in the fully connected layer. Therefore, the $i$ th output value of this layer can be expressed as follows:

$$
x_{o_{i}}=f\left(\sum_{j=1}^{d} x_{F_{j}} w_{o_{i} j}+b_{o_{i}}\right)
$$

\subsection{The method for new design}

Fig. 4 shows the flowchart of the proposed method. The procedure is performed as follows:

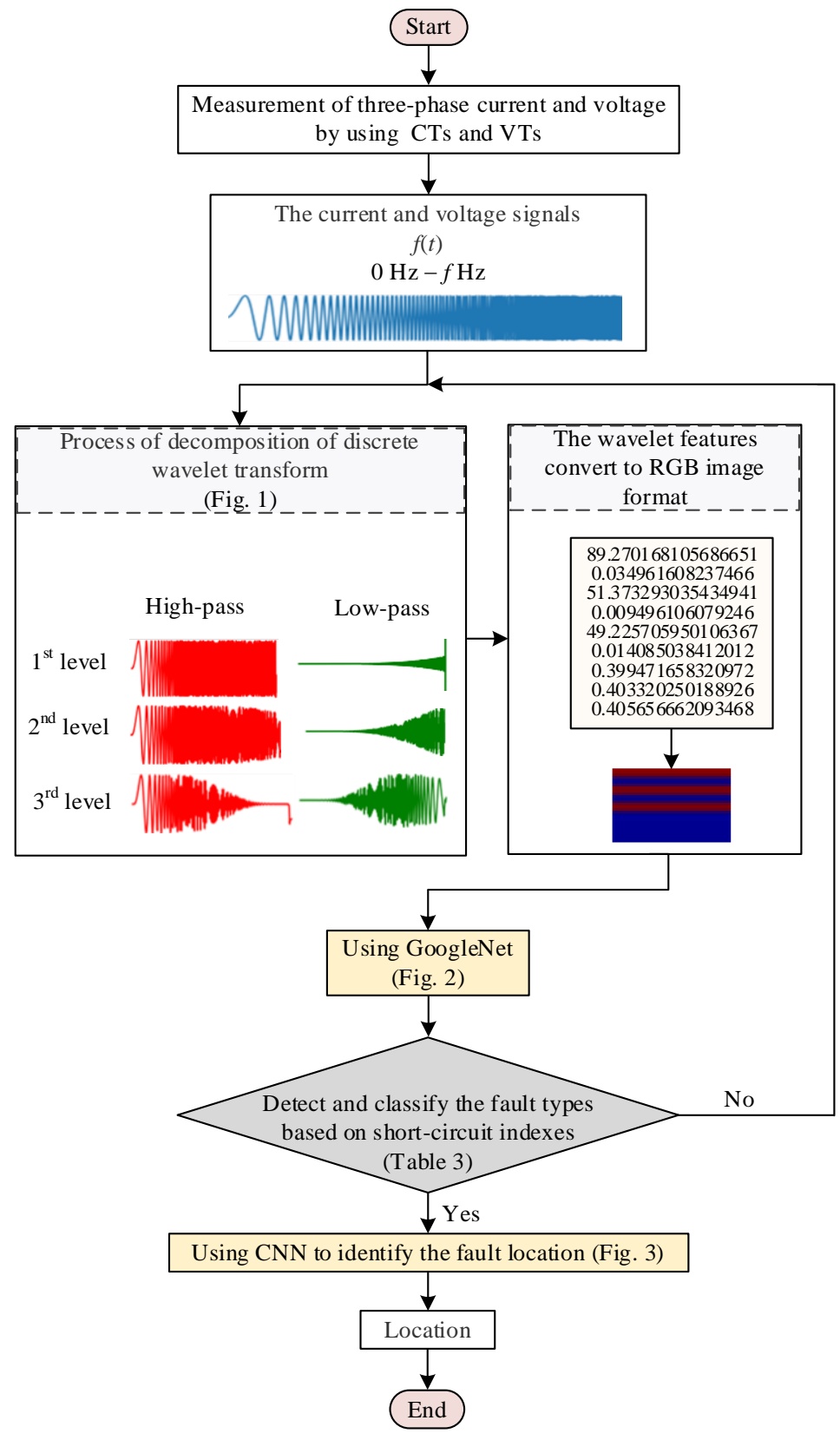

Fig. 4 The flow chart of the proposed method 
Step 1: Start

Step 2: The system's abnormal data including three-phase current and voltage through the Voltage Transformer (VT) and Current Transformer (CT) are loaded to implememt the next step.

Step 3: The raw fault signals with frequency from $0 \mathrm{~Hz}$ to $f \mathrm{~Hz}$ are collected from seven signals including currents and voltages of phases and neutral current.

Step 4: DWT-MRA is applied to extract features from the current or voltage signals on the transmission line as demonstrated in Fig. 1. Each signal is dissociated into a feature vector including four elements. For this step, the current and voltage signals of two cycles (including one pre- and post-fault cycle) are decomposed into three detail and approximate coefficients with the aid of Daubechies (db4) mother wavelet through a three-level decomposition. Eleven voltage and current coefficients, including four $3^{\text {rd }}$ order approximate components of four three-phase and neutral current, four $1^{\text {st }}$ order detailed comments of four single-phase and neutral current, and three $3^{\text {rd }}$ order approximate components of voltage signal, are used to analyze and characterize the fault.

Step 5: The wavelet feature components of each fault to the energy level of the signal are calculated based on Parseval's theorem and converted to the RGB image having the size of $224 \times 224 \times 3$.

Step 6: The GoogLeNet model as shown in Fig. 2 is used to train and test images with the purpose to achieve the classification of different fault states.

Step 7: The fault types are detected and classified based on the short-circuit indexes as shown in Table 3 . If step 7 is satisfied, then go to step 8 . If step 7 is not satisfied, then repeat step 3.

Step 8: The classified fault is located using CNN as shown in Fig. 3.

Step 9: End

Table 3 Short-circuit indexes

\begin{tabular}{|c|c|c|c|c|c|}
\hline \multirow{2}{*}{ No. } & Types of the short-circuit & \multicolumn{4}{|c|}{ Short-circuit indexes } \\
\cline { 3 - 6 } & & LLLG & LL & LLG & SLG \\
\hline 1 & Single Line-to-Ground (SLG) fault & - & - & - & 0.99 \\
\hline 2 & Line-to-Line (LL) fault & - & 0.51 & - & - \\
\hline 3 & Double Line-to-Ground (LLG) fault & - & - & 0.87 & - \\
\hline 3 & Three Line-to-Ground (LLLG) & 0.75 & - & - & - \\
\hline
\end{tabular}

\section{Implementation and Evaluation}

The system as shown in Fig. 5 is considered to test and verify the effectiveness of the proposed method. The $220 \mathrm{kV}$ transmission line with a length of $200 \mathrm{~km}$ is interconnected between two $\mathrm{B}_{1}$ and $\mathrm{B}_{2}$ buses. The power source is connected to $\mathrm{B}_{1}$ through the transformer $\mathrm{T} 1$, and the three-phase impedance load is connected to $\mathrm{B}_{2}$ through the transformer T2. The parameters of this system are listed in Table 4. The transmission line is modeled as the distributed parameter model. The system is simulated by using Matlab software. The frequency of the electrical system is $50 \mathrm{~Hz}$. The voltage and current signals with the sampling frequency are $2.4 \mathrm{kHz}$, which are equivalent to 48 samples per cycle.

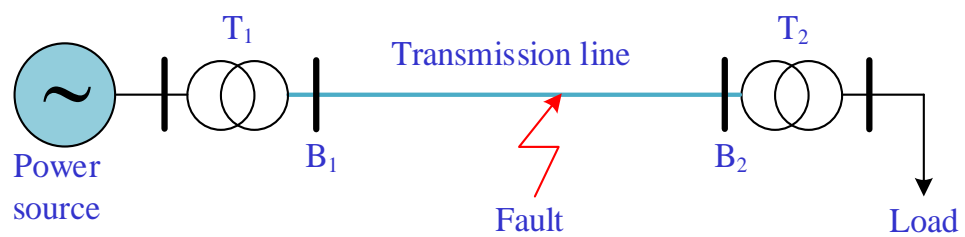

Fig. 5 The simple two-bus transmission line system 
In this case, 50 fault locations are considered on the transmission line with a length of $200 \mathrm{~km}$ starting from $4 \mathrm{~km}$ to 196 $\mathrm{km}$ with the step size of $4 \mathrm{~km}$, and are applied to the types of faults. The considered faults, i.e., SLG, LL, LLG, and LLLG, are applied to each location fault. To generate the training and test data for the combined network between GoogleNet and wavelet technology, the fault resistance value is randomly selected from $0 \Omega$ to $15 \Omega$ and the pre-fault loading condition changes in a range of $\pm 5 \%$. To detect and classify faults, 450 faulted scenarios for 10 types of faults are considered the inputs of the WT extracted features. To evaluate the effectiveness of the proposed algorithm, fault detection is performed on random locations of the transmission line by the condition of the change of the pre-fault loading and fault impedance. Similarly, this technique collects features for 450 non-faulted scenarios considering the conditions of varying loading. The analyzed results of approximate and detail coefficients of wavelet at the first and third levels of currents and voltages for SLG, LL, LLG, and LLLG faults are shown in Figs. 6, 7, 8, and 9, respectively. This analysis is based on three-phase current and voltage signals in one pre- and post-cycle into three detail and three approximate coefficients with the aid of Daubechies (db4) mother wavelet through a three-level decomposition.

The SLG fault on phase A has been simulated at the $3^{\text {rd }}$ cycle. The DWT-based decomposition of the current and the voltage supplied by the generator is provided in Fig. 6. The high values of detail coefficients at first level decomposition as shown in Figs. 6 (c)-(f) detect the occurrence of SLG fault in the system. Figs. 6(a), (g), (h), and (i) represent the original and level 3 approximate coefficient voltage signals, respectively. The magnitude of voltage during the faulty condition is relatively high as compared to the healthy condition. Figs. 6(b), (j), (k), (l), and (m) represent the original and level 3 approximate coefficients of phases and neutral current signals, respectively. The magnitude of current during the faulty condition is relatively high as compared to the healthy condition.

Table 4 Parameter details of the tested power transmission line

\begin{tabular}{|c|c|}
\hline Equipment & Details \\
\hline Transformer T1 & 210 MVA, 13.8/220 kV, Yg-D \\
\hline Transformer T2 & 210 MVA, 220/110 kV, D-Yg \\
\hline Load & $190 \mathrm{MW}, 100 \mathrm{MVAr}$ (inductive) \\
\hline Transmission line & $200 \mathrm{~km}$ \\
\hline Frequency & $50 \mathrm{~Hz}$ \\
\hline Fault impedance & $0-15 \Omega$ \\
\hline Load variation & $\pm 5 \%$ \\
\hline
\end{tabular}

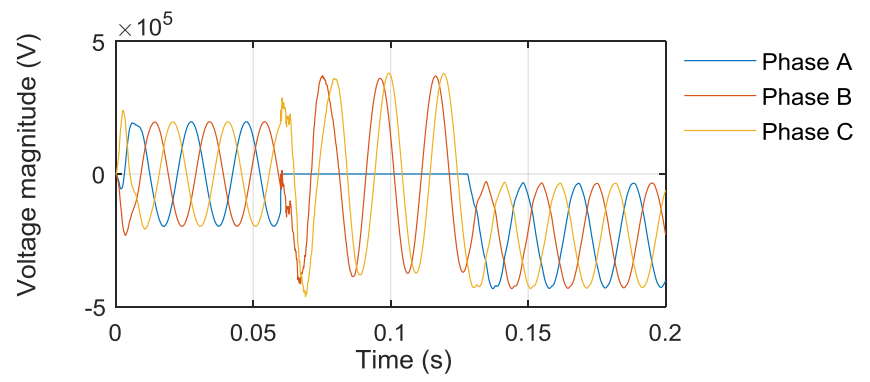

(a) Three-phase voltage

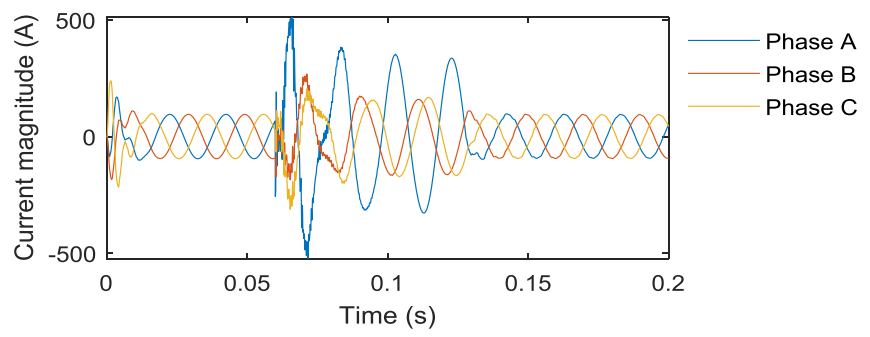

(b) Three-phase current

Fig. 6 The detail and approximate coefficients of the SLG fault case 


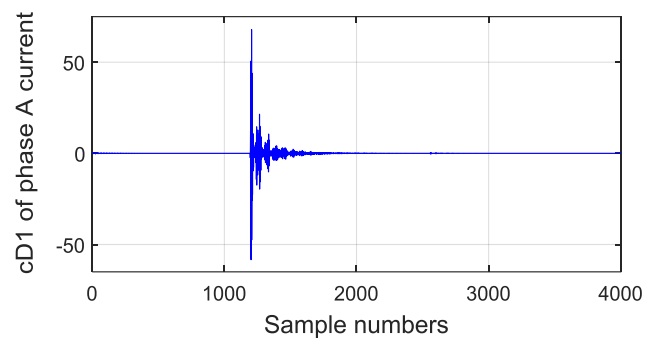

(c) Detail coefficient 1 of the phase A voltage

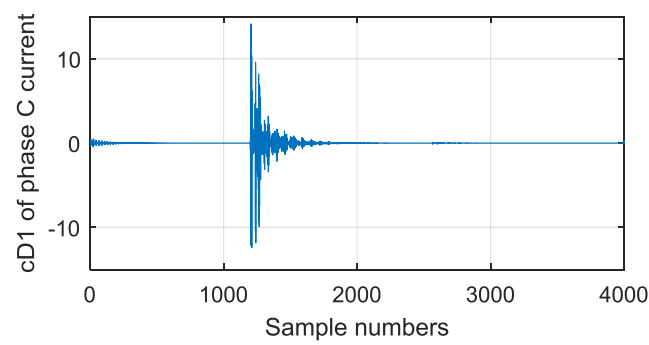

(e) Detail coefficient 1 of the neutral current

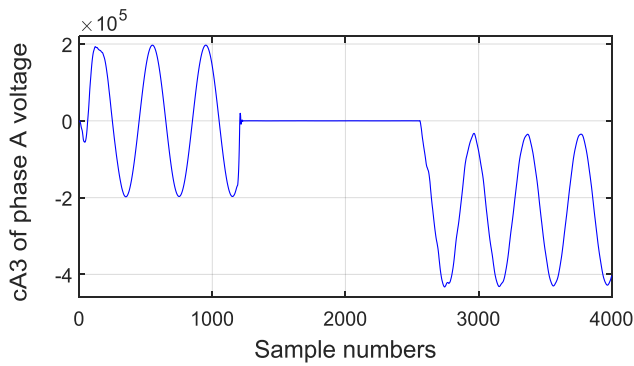

(g) Approximate coefficient 3 of the phase A voltage

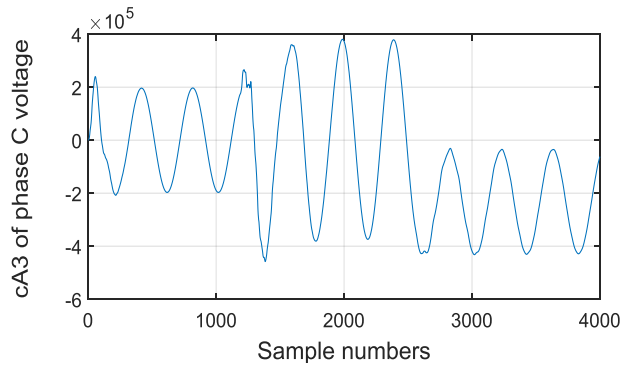

(i) Approximate coefficient 3 of the phase $\mathrm{C}$ voltage

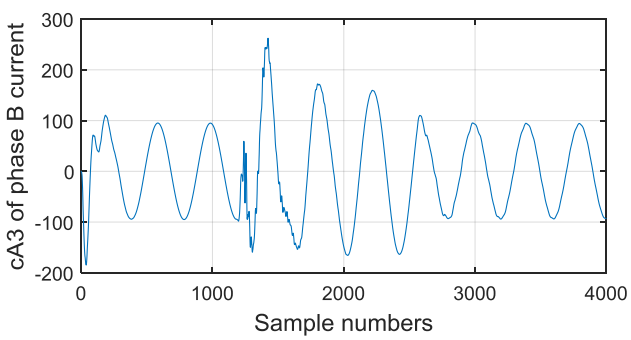

(k) Approximate coefficient 3 of the phase B current

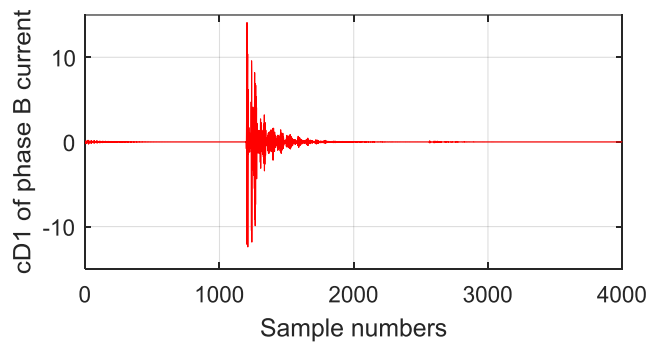

(d) Detail coefficient 1 of the phase B current

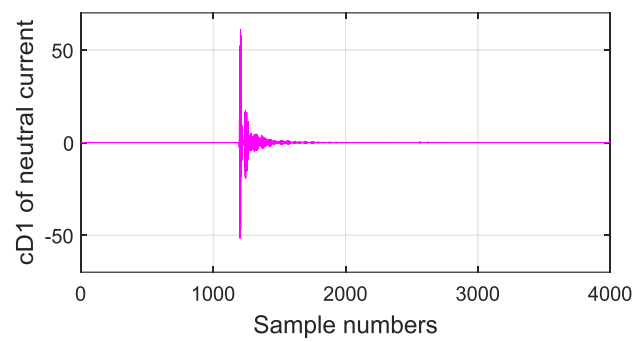

(f) Detail coefficient 1 of the neutral current

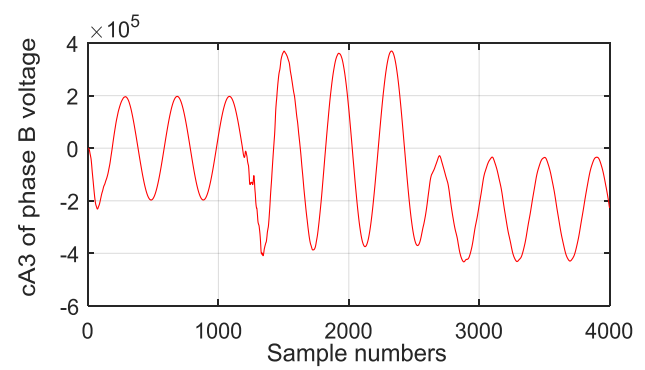

(h) Approximate coefficient 3 of the phase B voltage

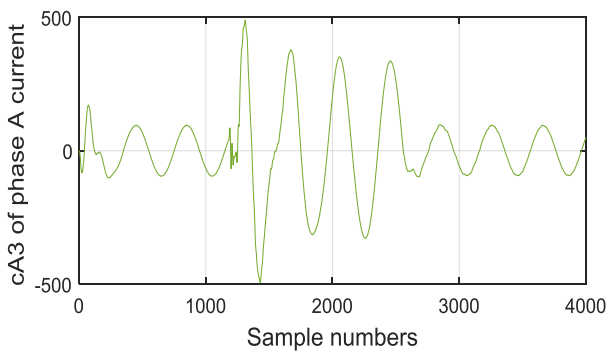

(j) Approximate coefficient 3 of the phase A current

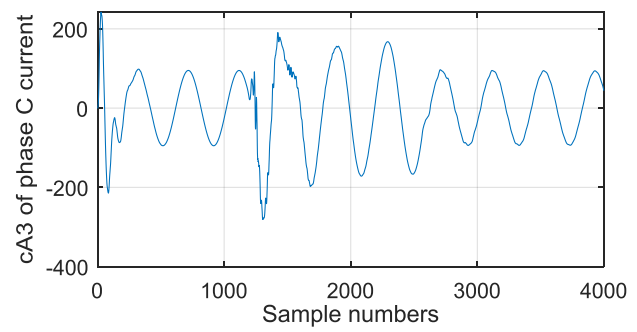

(1) Approximate coefficient 3 of the phase A current

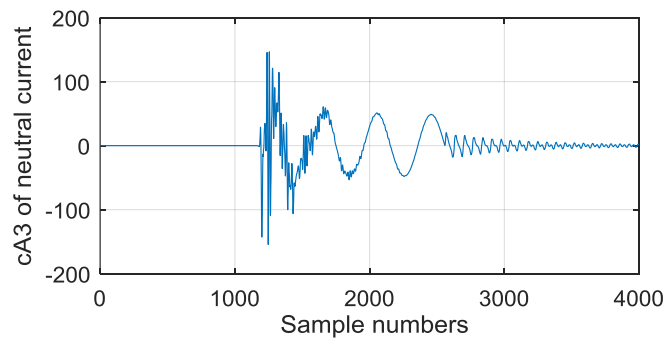

(m) Approximate coefficient 3 of the neutral current

Fig. 6 The detail and approximate coefficients of the SLG fault case (continued) 
The LL fault on phases A and B has been simulated at the $3^{\text {rd }}$ cycle by simultaneously grounding the phases A and B. The DWT-based decomposition of the current and the voltage supplied by the generator is provided in Fig. 7. The high values of detail coefficients at first level decomposition as shown in Figs. 7 (c), (d), (e), and (h) detect the occurrence of LL fault in the system. Figs. 7(a) and (f) represent the original and level 3 approximate coefficient voltage signals, respectively. The magnitude of voltage during the faulty condition is relatively high as compared to the healthy condition. Figs. 7(b), (g), and (h) represent the original and level 3 approximate coefficients and neutral current signals, respectively. The magnitude of current during the faulty condition is relatively high as compared to the healthy condition.

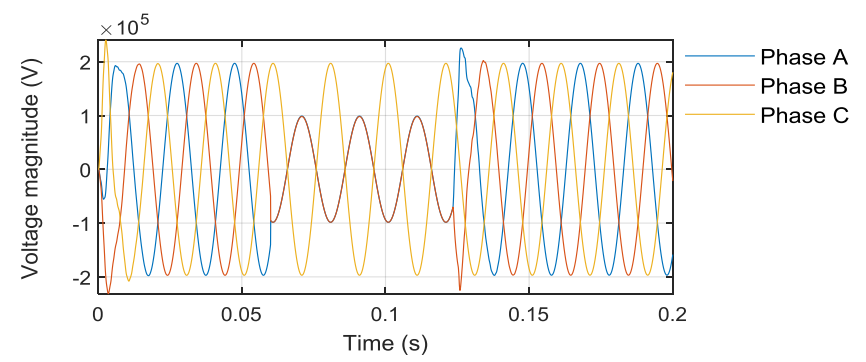

(a) Three-phase voltage

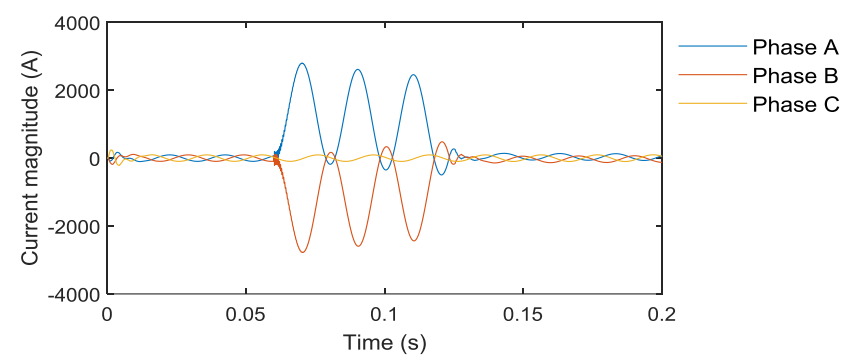

(b) Three-phase current

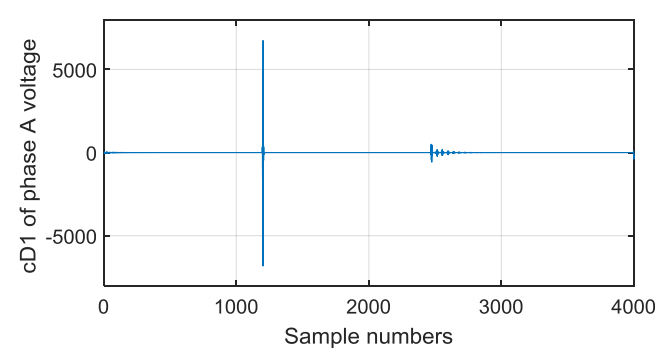

(c) Detail coefficient 1 of the phase A voltage

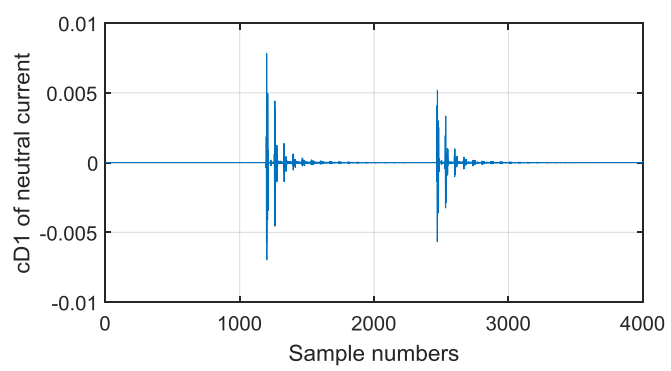

(e) Detail coefficient 1 of the neutral current

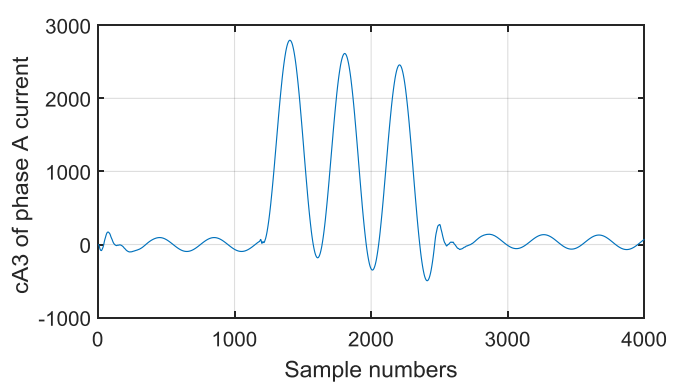

(g) Approximate coefficient 3 of the phase A current

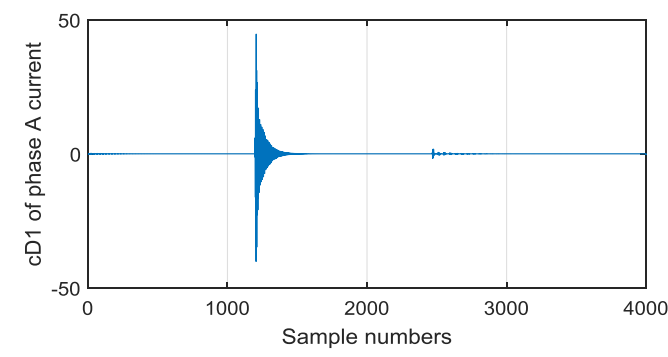

(d) Detail coefficient 1 of the phase A current

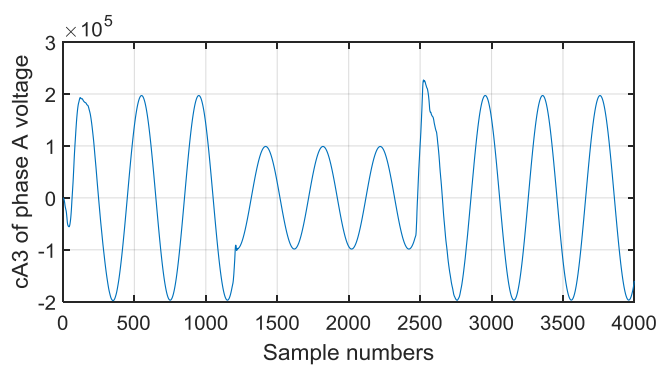

(f) Approximate coefficient 3 of the phase A voltage

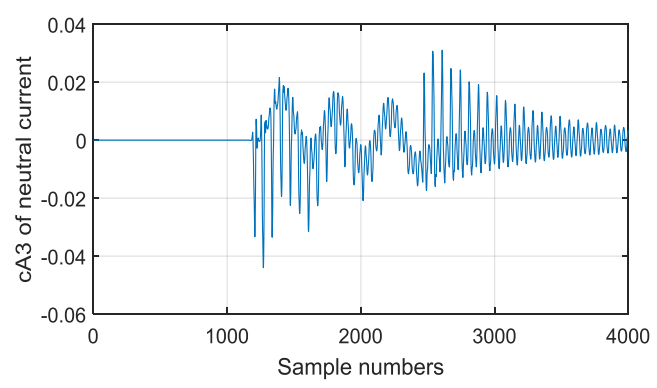

(h) Approximate coefficient 1 of the neutral current

Fig. 7 The detail and approximate coefficients of the LL fault case 
The LLG fault on phases A and B has been simulated at the $3^{\text {rd }}$ cycle by simultaneously circuiting the phase A and B. The DWT-based decomposition of the current and the voltage supplied by the generator is provided in Fig. 8. The high values of detail coefficients at first level decomposition as shown in Figs. 8 (c), (d), (e), and (h) detect the occurrence of LLG fault in the system. Figs. 8(a) and (f) represent the original and level 3 approximate coefficient voltage signals, respectively. The magnitude of voltage during the faulty condition is relatively high as compared to the healthy condition. Figs. 8(b), (g), and (h) represent the original and level 3 approximate coefficients and neutral current signals, respectively. The magnitude of current during the faulty condition is relatively high as compared to the healthy condition.

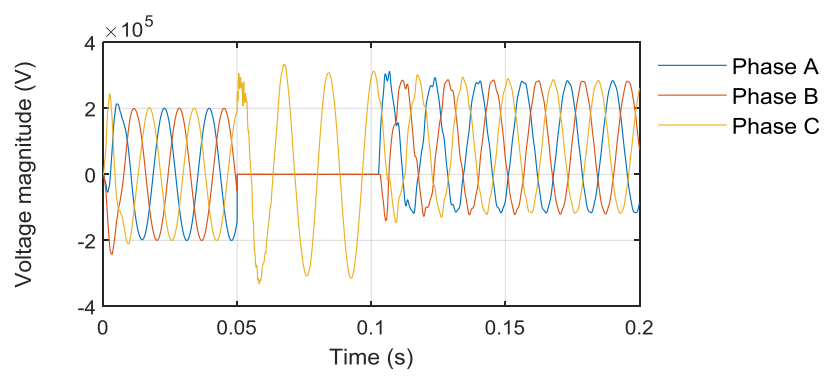

(a) Three-phase voltage

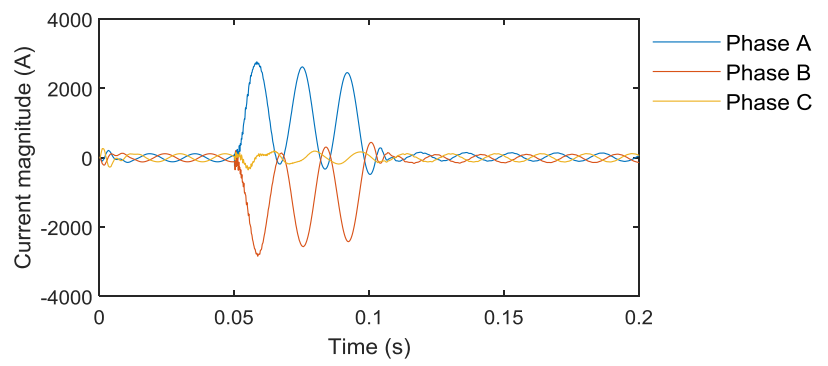

(b) Three-phase current

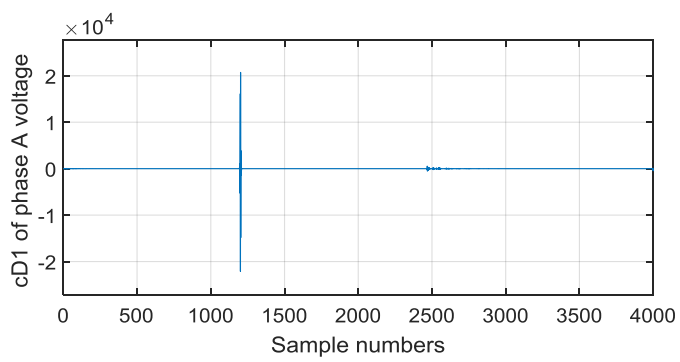

(c) Detail coefficient 1 of the phase A voltage

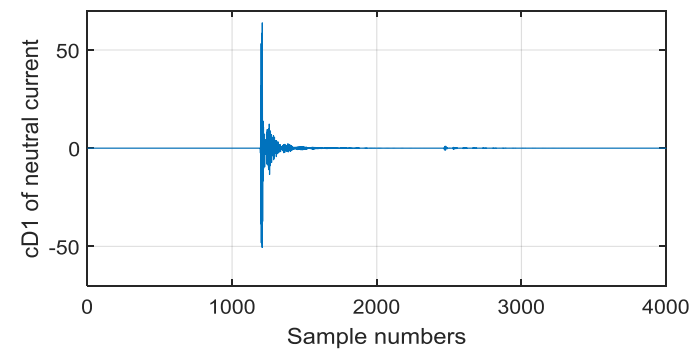

(e) Detail coefficient 1 of the neutral current

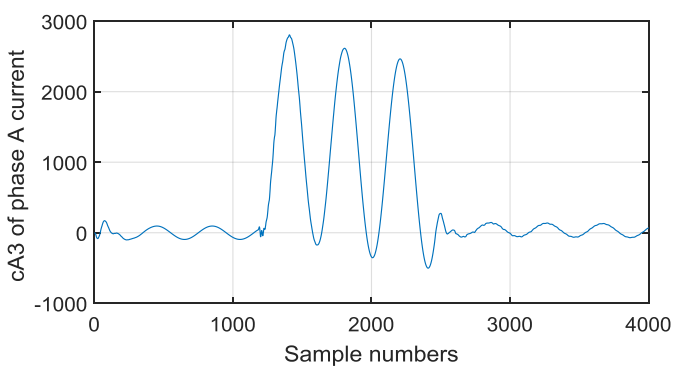

(g) Approximate coefficient 3 of the phase A current

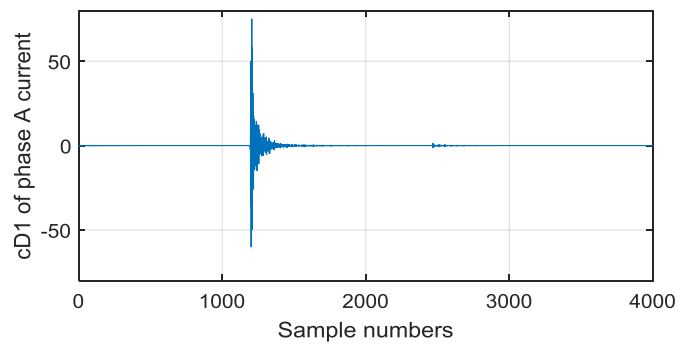

(d) Detail coefficient 1 of the phase A current

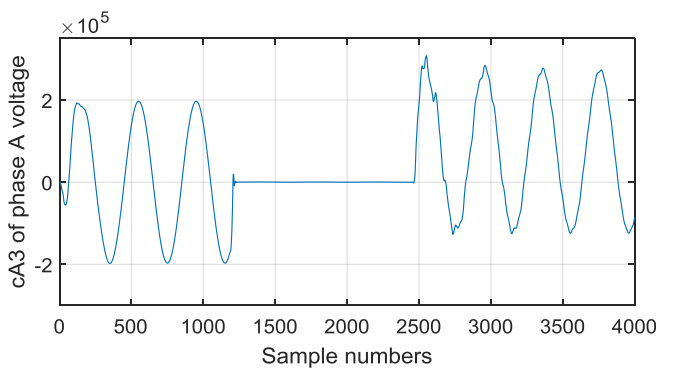

(f) Approximate coefficient 3 of the phase A voltage

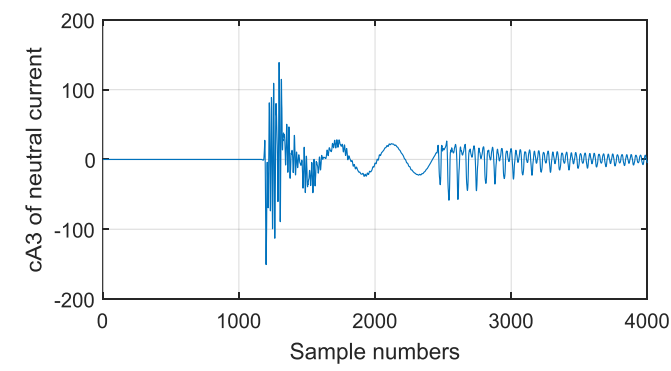

(h) Approximate coefficient 1 of the neutral current

Fig. 8 The detail and approximate coefficients of the LLG fault case 
The LLLG fault has been simulated at the $3^{\text {rd }}$ cycle by simultaneously circuiting the phase A, B, and C. The DWT-based decomposition of the current and the voltage supplied by the generator is provided in Fig. 9. The high values of detail coefficients at first level decomposition as shown in Figs. 9 (c), (d), (e), and (h) detect the occurrence of LLLG fault in the system. Figs. 9 (a) and (f) represent the original and level 3 approximate coefficient voltage signals, respectively. The magnitude of voltage during the faulty condition is relatively high as compared to the healthy condition. Figs. 9(b), (g), and (h) represent the original and level 3 approximate coefficients and neutral current signals, respectively. The magnitude of current during the faulty condition is relatively high as compared to the healthy condition.

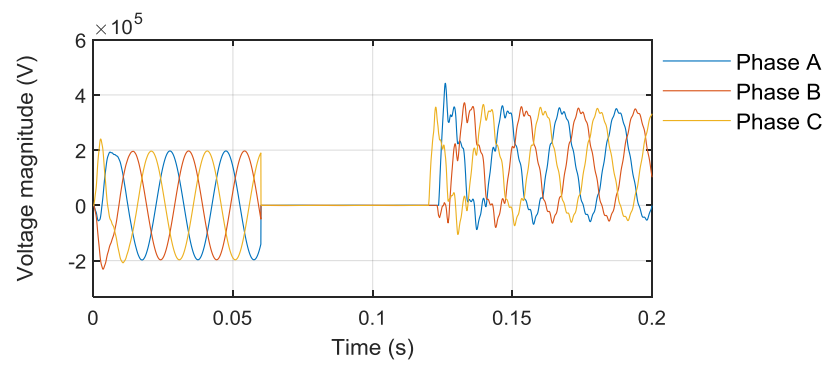

(a) Three-phase voltage

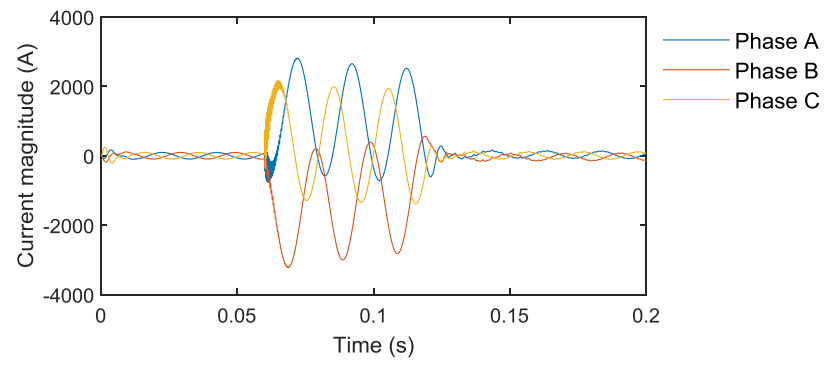

(b) Three-phase current

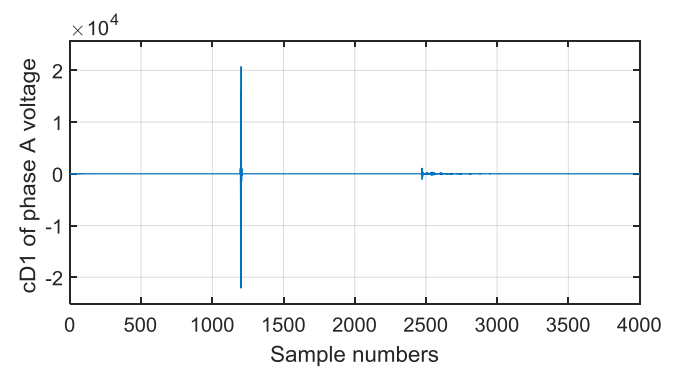

(c) Detail coefficient 1 of the phase A voltage

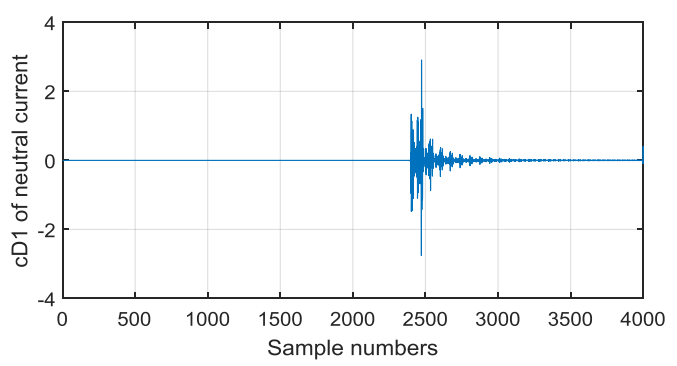

(e) Detail coefficient 1 of the neutral current

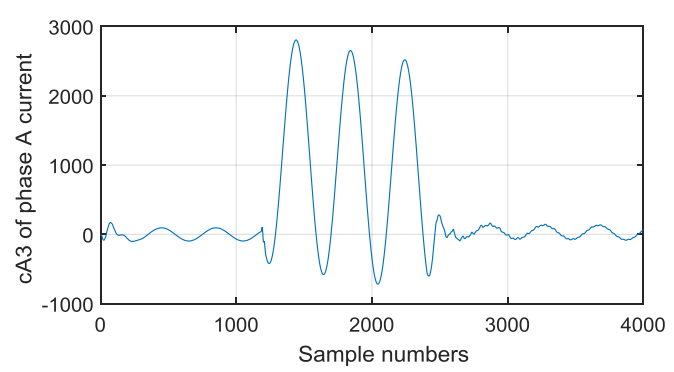

(g) Approximate coefficient 3 of the phase A current

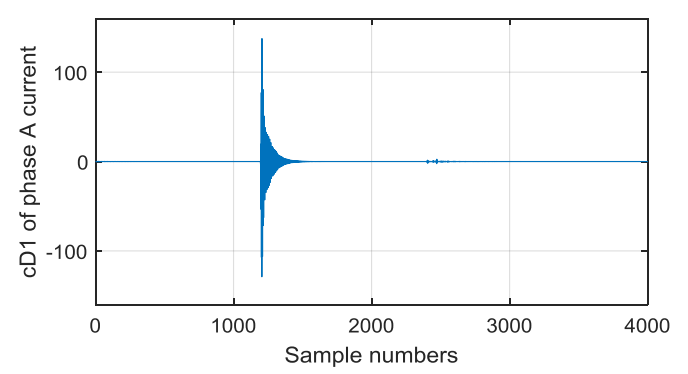

(d) Detail coefficient 1 of the phase A current

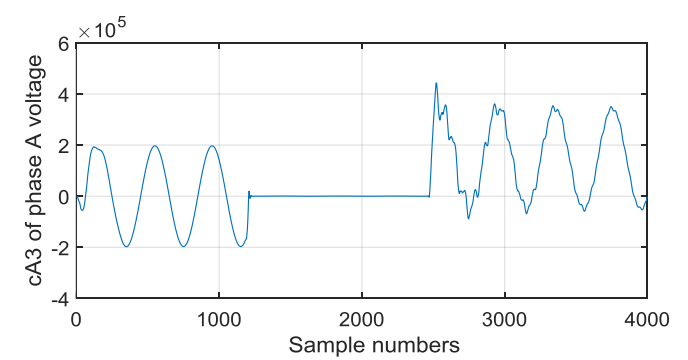

(f) Approximate coefficient 3 of the phase A voltage

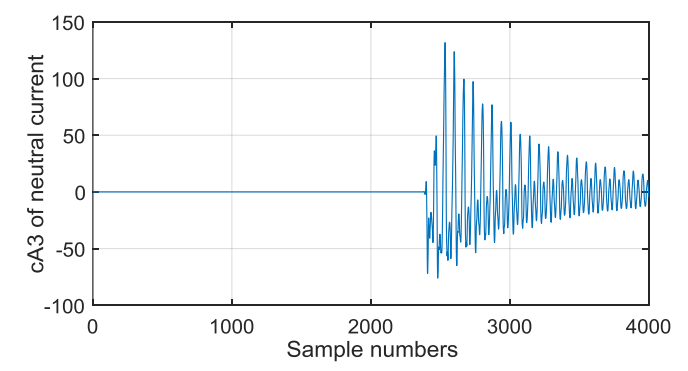

(h) Approximate coefficient 1 of the neutral current

Fig. 9 The detail and approximate coefficients of the LLLG fault case 
Eleven voltage and current coefficients, including four $3^{\text {rd }}$ order approximate components of four three-phase and neutral current, four $1^{\text {st }}$ order detailed comments of four single-phase and neutral current, and three $3^{\text {rd }}$ order approximate components of voltage signal, are used to analyze and characterize the fault. The data extracted from the above eleven characteristic wavelets are calculated to the energy level, and the data of teen fault types are calculated based on Parseval's theorem and converted to the RGB image having the size of $224 \times 224 \times 3$. Fig. 10 shows the RGB image of LLLG, LLG, LL, and SLG faults that are obtained from the eleven featured components of the wavelet.

The data of 10 fault types are converted to 10 RGB image formats as the input of the GoogLeNet model. Fig. 11 shows the training results when using the GoogleNet network with 450 faulted scenarios at 49 locations on the $220 \mathrm{kV}$ transmission line with a length of $200 \mathrm{~km}$. Table 5 is the accurate ratio results of training GoogleNet. Fig. 11 and Table 5 show that after the training time of 5 minutes and 3 seconds, the number of iteration is 40, the number of epoch is 20, and the obtained result is $100 \%$ accurate for the SLG fault case. Fig 12 (a) shows the testing result based on each data of this fault type when using the GoogleNet network. The obtained result is exactly for classifying the form of SLG fault, and the output data feature corresponding to the considered fault location is 0.995 .

Similarly, Figs. 12 (b), (c), and (d) show the testing result based on each data of LL, LLG, and LLLG faults when using the GoogleNet network. Observing from this figure, the obtained result is exactly for classifying the form of LL, LLG, and LLLG faults, and the output data feature corresponding to the considered fault location are $0.7512,0.5169$, and 0.8742 , respectively.

\begin{tabular}{|c|c|c|c|}
\hline $\begin{array}{c}\text { Eleven featured } \\
\text { components } \\
\text { of wavelet }\end{array}$ & RGB image & $\begin{array}{c}\text { Eleven featured } \\
\text { components } \\
\text { of wavelet }\end{array}$ & RGB image \\
\hline \multicolumn{2}{|c|}{ LLLG fault } & \multicolumn{2}{|c|}{ LLG fault } \\
\hline 3.238606563886608 & & 3.296538351665102 & \\
\hline 0.023803439770879 & & 0.010910527870775 & \\
\hline 3.775082416306386 & & 3.263021840146756 & \\
\hline 0.008492275993748 & & 0.006246329955996 & \\
\hline 2.569740496679839 & & 0.323046891204528 & \\
\hline 0.032275330373190 & & 0.003214583506036 & \\
\hline 0.074524570669652 & & 0.066346468565089 & \\
\hline 0.000477381350003 & & 0.009656086756668 & \\
\hline 0.679451905560334 & & 0.572634788701199 & \\
\hline 0.682172860734557 & & 0.572408623953854 & \\
\hline 0.6688887326904826 & & 0.786176922858430 & \\
\hline \multicolumn{2}{|c|}{ LL fault } & \multicolumn{2}{|c|}{ SLG fault } \\
\hline 3.287512700347871 & & 0.595018315943588 & \\
\hline 0.007678461669207 & & 0.009945768353923 & \\
\hline 3.268005076837985 & & 0.337635492728258 & \\
\hline 0.007676283363128 & & 0.002846534238161 & \\
\hline 0.274710467772305 & & 0.361750447617142 & \\
\hline 0.000114787471117 & & 0.002849826567672 & \\
\hline 0.000029160395831 & & 0.094019243908815 & \\
\hline 0.000001402853392 & & 0.009531795875448 & \\
\hline 0.558107940602884 & & 0.830152577181211 & \\
\hline 0.553148650476941 & & 1.046783682310400 & \\
\hline 0.631255640157050 & & 1.097386771455402 & \\
\hline
\end{tabular}

Fig. 10 The featured comments of wavelet and RGB images of the fault types

Table 5 The accurate ratio results of training GoogleNet for SLG fault

\begin{tabular}{|c|c|c|c|c|c|c|c|}
\hline Epoch & Iteration & $\begin{array}{c}\text { Time } \\
\text { elapsed }\end{array}$ & $\begin{array}{c}\text { Mini-batch } \\
\text { accuracy }\end{array}$ & $\begin{array}{c}\text { Validation } \\
\text { accuracy }\end{array}$ & $\begin{array}{c}\text { Mini-batch } \\
\text { loss }\end{array}$ & $\begin{array}{c}\text { Validation } \\
\text { loss }\end{array}$ & $\begin{array}{c}\text { Base } \\
\text { leaning rate }\end{array}$ \\
\hline 1 & 1 & $00: 00: 12$ & $26.67 \%$ & $25.00 \%$ & 2.2122 & 1.6795 & $1.0000 \mathrm{e}-04$ \\
\hline 5 & 10 & $00: 01: 22$ & $40.00 \%$ & $50.00 \%$ & 1.7446 & 1.1212 & $1.0000 \mathrm{e}-04$ \\
\hline 10 & 20 & $00: 02: 38$ & $66.67 \%$ & $50.00 \%$ & 0.9892 & 0.8711 & $1.0000 \mathrm{e}-04$ \\
\hline 15 & 30 & $00: 03: 51$ & $60 \%$ & $75.00 \%$ & 0.8442 & 0.7838 & $1.0000 \mathrm{e}-04$ \\
\hline 20 & 40 & $00: 05: 03$ & $80.00 \%$ & $100.00 \%$ & 0.5734 & 0.7072 & $1.0000 \mathrm{e}-04$ \\
\hline
\end{tabular}


The obtained result after classifying is used for locating the fault by CNN. The obtained results for identifying the SLG fault are shown in Table 6 and the obtained training result is shown in Fig. 11. Table 6 shows that the average error about location comparing to the actual is $0.215 \mathrm{~km}$.

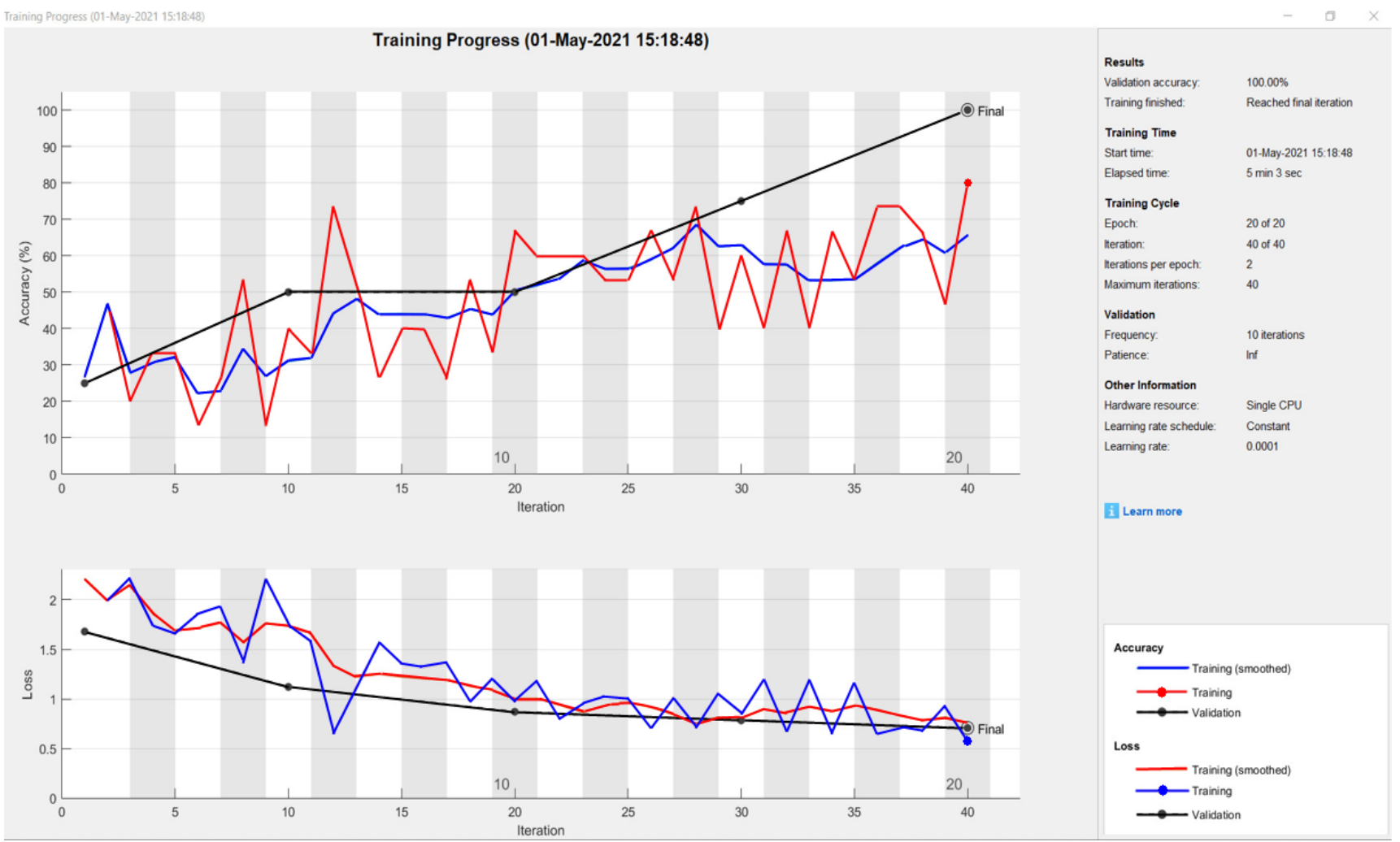

Fig. 11 The GoogleNet training result of SLG fault

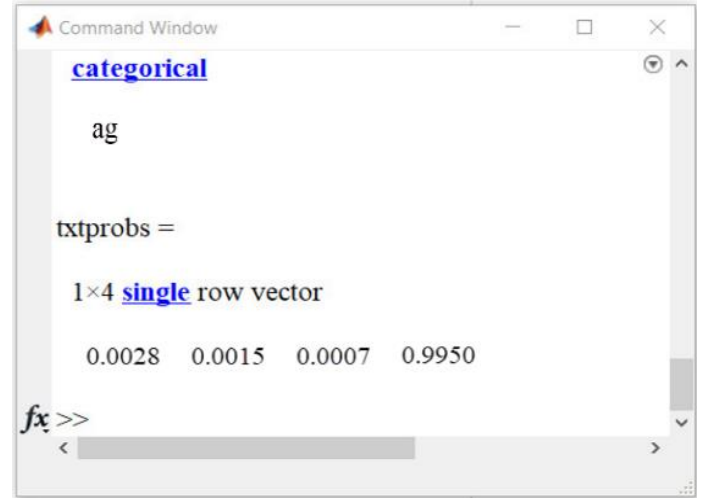

(a) Command window: SLG fault

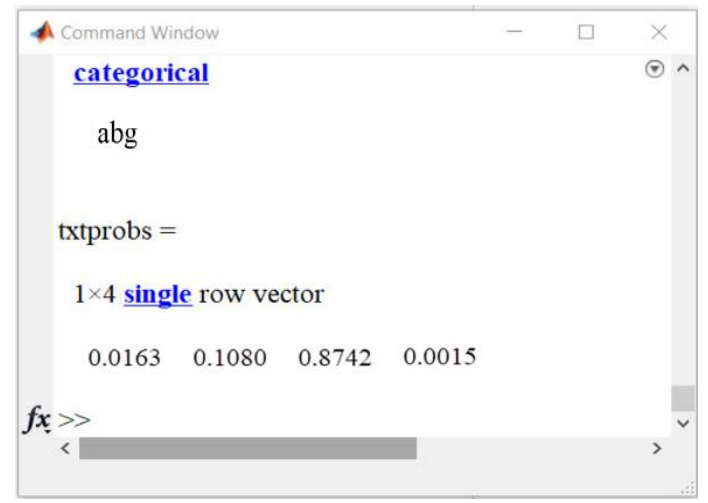

(c) Command window: LLG fault

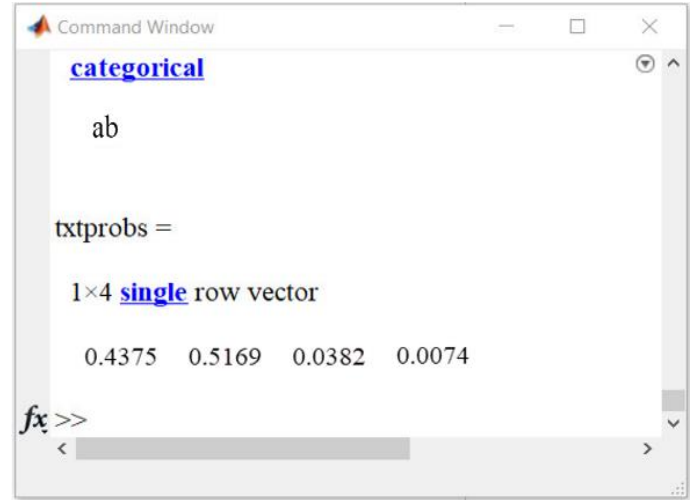

(b) Command window: LL fault

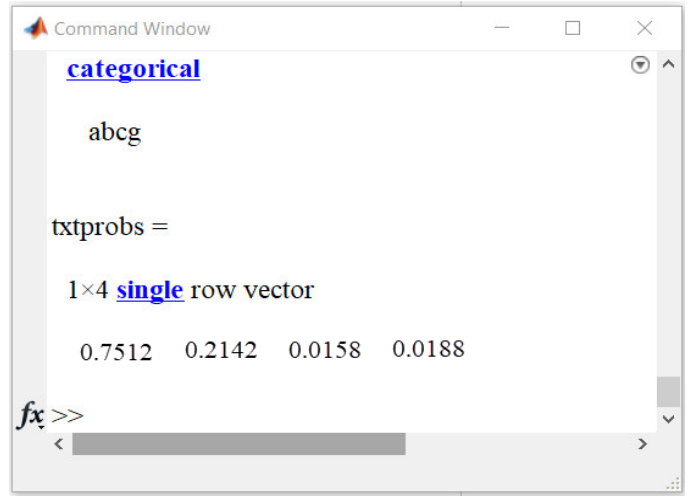

(d) Command window: LLLG fault

Fig. 12 The predicted result using GoogLeNet 
Table 6 The located result for SLG fault

\begin{tabular}{|c|c|c|}
\hline \multicolumn{2}{|c|}{ Distance $(\mathrm{km})$} & \multirow{2}{*}{$\begin{array}{c}\text { Error } \\
(\mathrm{km})\end{array}$} \\
\cline { 1 - 2 } Actual & Proposed & 0.006 \\
\hline 21 & 20.994 & 0.005 \\
\hline 28 & 27.995 & 0.016 \\
\hline 35 & 34.984 & 0.004 \\
\hline 41 & 40.996 & 0.009 \\
\hline 47 & 46.991 & 0.011 \\
\hline 53 & 52.989 & 0.007 \\
\hline 59 & 58.993 & 0.009 \\
\hline 65 & 64.991 & 0.008 \\
\hline 72 & 71.992 & 0.009 \\
\hline 79 & 78.991 & 0.098 \\
\hline 110 & 110.098 & 0.122 \\
\hline 130 & 130.122 & 0.280 \\
\hline 150 & 150.280 & 0.426 \\
\hline 170 & 169.574 & 0.331 \\
\hline 190 & 189.669 & Average error: 0.215 \\
\hline \multicolumn{3}{|c|}{} \\
\hline \multicolumn{2}{|c|}{}
\end{tabular}

\section{Conclusions}

In this study, a hybrid method is developed based on a combination of WT, GoogLeNet, and CNN to identify, classify, and locate the electrical fault on the transmission lines. Firstly, DWT-MRA is proposed to extract two cycles (pre-cycle and post-cycle) of three-phase fault current/voltage signals measured from the sending end, and decompose these signals into three detail and approximate coefficients with the aid of Daubechies (db4) mother wavelet through a three-level decomposition. Then, the WT feature components of each fault are calculated to the signal energy level based on Parseval's theorem and converted to the RGB images having the size of $224 \times 224 \times 3$. These RGB images are input into The GoogLeNet model for fault classification. Finally, the five-layer CNN is applied to locate the fault.

The proposed method is tested on the four-bus power system with a length of $220 \mathrm{~km}$ of the $220 \mathrm{kV}$ transmission line via time-domain simulation using Matlab software. Different fault types, including the symmetrical and unsymmetrical faults, are considered to conduct the simulation. In addition, the fault resistance from $0 \Omega$ to $15 \Omega$ and a range of $\pm 5 \%$ of changing load is carried out to simulate for case studies. The simulation results show that the proposed fault detection, classification, and location method is very reliable and simple, and has a fast processing time. Therefore, the proposed method is a useful tool for analyzing the system stability in the field of electricity.

\section{Acknowledgments}

This work belongs to the project grant No: T2020-27TĐ, which was supported by the Ho Chi Minh City University of Technology and Education, Ho Chi Minh City, Vietnam.

\section{Conflicts of Interest}

The authors declare no conflict of interest.

\section{References}

[1] L. V. Dai, D. D. Tung, and L. C. Quyen, "A Highly Relevant Method for Incorporation of Shunt Connected FACTS Device into Multi-Machine Power System to Dampen Electromechanical Oscillations,” Energies, vol. 10, no. 4, 482, April 2017. 
[2] L. V. Dai, D. D. Tung, T. L. T. Dong, and C. L. Quyen, "Improving Power System Stability with Gramian Matrix-Based Optimal Setting of a Single Series Facts Device: Feasibility Study in Vietnamese Power System,” Complexity, vol. 2017, 3014510, January 2017.

[3] Ravi, "Causes, Nature and Effect of Fault in Power in Power System," http://electricalarticle.com/causes-nature-effect-fault-power-system, December 26, 2019

[4] C. Cortes and V. Vapnik, "Support-Vector Networks," Machine Learning, vol. 20, no. 3, pp. 273-297, September 1995.

[5] U. B. Parikh, B. Das, and R. Maheshwari, "Fault Classification Technique for Series Compensated Transmission Line Using Support Vector Machine,” International Journal of Electrical Power Energy Systems, vol. 32, no. 6, pp. 629-636, July 2010.

[6] P. Ray and D. P. Mishra, "Support Vector Machine Based Fault Classification and Location of a Long Transmission Line," Engineering Science Technology, an International Journal, vol. 19, no. 3, pp. 1368-1380, September 2016.

[7] N. R. Babu and B. J. Mohan, "Fault Classification in Power Systems Using EMD and SVM," Ain Shams Engineering Journal, vol. 8, no. 2, pp. 103-111, June 2017.

[8] Y. Guo, K. Li, and X. Liu, "Fault Diagnosis for Power System Transmission Line Based on PCA and SVMs," International Conference on Intelligent Computing for Sustainable Energy and Environment, pp. 524-532, September 2012.

[9] V. Malathi and N. Marimuthu, "Multi-Class Support Vector Machine Approach for Fault Classification in Power Transmission Line," IEEE International Conference on Sustainable Energy Technologies, pp. 67-71, November 2008.

[10] H. Livani and C. Y. Evrenosoğlu, "A Fault Classification Method in Power Systems Using DWT and SVM Classifier," IEEE PES Transmission and Distribution Conference and Exposition, pp. 1-5, May 2012.

[11] V. Ferreira, R. Zanghi, M. Fortes, G. Sotelo, R. Silva, J. Souza, et al., "A Survey on Intelligent System Application to Fault Diagnosis in Electric Power System Transmission Lines,” Electric Power Systems Research, vol. 136, pp. 135-153, July 2016.

[12] A. Prasad, J. B. Edward, and K. Ravi, “A Review on Fault Classification Methodologies in Power Transmission Systems: Part-I,” Journal of Electrical Systems, vol. 5, no. 1, pp. 48-60, May 2018.

[13] H. K. Zadeh and M. Aghaebrahimi, "A Novel Approach to Fault Classification and Fault Location for Medium Voltage Cables Based on Artificial Neural Network," International Journal of Computational Intelligence, vol. 2, no. 1, pp. 1304-2386, 2005.

[14] S. Ekici, S. Yildirim, and M. Poyraz, "Energy and Entropy-Based Feature Extraction for Locating Fault on Transmission Lines by Using Neural Network and Wavelet Packet Decomposition," Expert Systems with Applications, vol. 34, no. 4, pp. 2937-2944, May 2008.

[15] Y. S. Rao, G. R. Kumar, and G. K. Rao, “A New Approach for Classification of Fault in Transmission Line with Combination of Wavelet Multi Resolution Analysis and Neural Networks," International Journal of Power Electronics, vol. 8, no. 1, 505, 2017.

[16] M. Saradarzadeh and M. S. Pasand, "An Accurate Fuzzy Logic-Based Fault Classification Algorithm Using Voltage and Current Phase Sequence Components,” International Transactions on Electrical Energy Systems, vol. 25, no. 10, pp. 2275-2288, October 2015.

[17] A. Prasad, J. B. Edward, C. S. Roy, G. Divyansh, and A. Kumar, "Classification of Faults in Power Transmission Lines Using Fuzzy-Logic Technique,” Indian Journal of Science, vol. 8, no. 30, pp. 1-6, 2015.

[18] S. Adhikari, N. Sinha, and T. Dorendrajit, "Fuzzy Logic Based On-Line Fault Detection and Classification in Transmission Line,” SpringerPlus, vol. 5, no. 1, pp. 1-14, July 2016.

[19] D. Thukaram, H. Khincha, and H. Vijaynarasimha, "Artificial Neural Network and Support Vector Machine Approach for Locating Faults in Radial Distribution Systems,” IEEE Transactions on Power Delivery, vol. 20, no. 2, pp. 710-721, April 2005.

[20] P. Nonyane, “The Application of Artificial Neural Networks to Transmission Line Fault Detection and Diagnosis,” Ph.D. dissertation, University of South Africa, Pretoria, 2016.

[21] M. Farshad and J. Sadeh, "Accurate Single-Phase Fault-Location Method for Transmission Lines Based on K-Nearest Neighbor Algorithm Using One-End Voltage,” IEEE Transactions on Power Delivery, vol. 27, no. 4, pp. 2360-2367, September 2012.

[22] P. Ray, "Fast and Accurate Fault Location by Extreme Learning Machine in a Series Compensated Transmission Line," Power and Energy Systems: Towards Sustainable Energy, pp. 1-6, March 2014.

[23] K. Hosseini, "Short Circuit Fault Classification and Location in Transmission Lines Using a Combination of Wavelet Transform and Support Vector Machines," International Journal on Electrical Engineering Informatics, vol. 7, no. 2, 353, June 2015. 
[24] E. U. Haq, H. Jianjun, K. Li, F. Ahmad, D. Banjerdpongchai, and T. Zhang, "Improved Performance of Detection and Classification of 3-Phase Transmission Line Faults Based on Discrete Wavelet Transform and Double-Channel Extreme Learning Machine,” Electrical Engineering, vol. 103, no. 2, pp. 953-963, April 2021.

[25] P. Ray and D. Mishra, "Application of Extreme Learning Machine for Underground Cable Fault Location,” International Transactions on Electrical Energy Systems, vol. 25, no. 12, pp. 3227-3247, December 2015.

[26] P. Ray, D. P. Mishra, K. Dey, and P. Mishra, "Fault Detection and Classification of a Transmission Line Using Discrete Wavelet Transform \& Artificial Neural Network," International Conference on Information Technology, pp. 178-183, December 2017.

[27] P. Ray, D. P. Mishra, and S. Mohaptra, "Fault Classification of a Transmission Line Using Wavelet Transform \& Fuzzy Logic,” 1st International Conference on Power Electronics, Intelligent Control, and Energy Systems, pp. 1-6, July 2016.

[28] C. K. Jung, K. H. Kim, J. B. Lee, and B. Klöckl, "Wavelet and Neuro-Fuzzy Based Fault Location for Combined Transmission Systems,” International Journal of Electrical Power and Energy Systems, vol. 29, no. 6, pp. 445-454, July 2007.

[29] M. Shafiullah, M. A. Abido, and Z. A. Hamouz, "Wavelet-Based Extreme Learning Machine for Distribution Grid Fault Location," IET Generation, Transmission, and Distribution, vol. 11, no. 17, pp. 4256-4263, November 2017.

[30] S. Thukral, O. P. Mahela, and B. Kumar, "Detection of Transmission Line Faults in the Presence of Wind Energy Power Generation Source Using Stockwell's Transform," International Conference on Issues and Challenges in Intelligent Computing Techniques, pp. 1-6, September 2019.

[31] D. P. Mishra and P. Ray, "Fault Detection, Location and Classification of a Transmission Line," Neural Computing and Applications, vol. 30, no. 5, pp. 1377-1424, September 2018.

[32] P. Malla, W. Coburn, K. Keegan, and X. H. Yu, "Power System Fault Detection and Classification Using Wavelet Transform and Artificial Neural Networks,” International Symp. on Neural Networks, pp. 266-272, July 2019.

[33] G. Cao, K. Zhang, K. Zhou, H. Pan, Y. Xu, and J. Liu, “A Feature Transferring Fault Diagnosis Based on WPDR, FSWT and GoogLeNet," IEEE International Instrumentation and Measurement Technology Conference, pp. 1-6, May 2020.

[34] M. F. Guo, N. C. Yang, and W. F. Chen, "Deep-Learning-Based Fault Classification Using Hilbert-Huang Transform and Convolutional Neural Network in Power Distribution Systems,” IEEE Sensors Journal, vol. 19, no. 16, pp. 6905-6913, April 2019.

[35] T. M. Lai, L. A. Snider, E. Lo, and D. Sutanto, "High-Impedance Fault Detection Using Discrete Wavelet Transform and Frequency Range and RMS Conversion,” IEEE Transactions on Power Delivery, vol. 20, no. 1, pp. 397-407, February 2005.

[36] S. G. Mallat, “A Theory for Multiresolution Signal Decomposition: The Wavelet Representation,” IEEE Transactions on Pattern Analysis and Machine Intelligence, vol. 11, no. 7, pp. 674-693, July 1989.

[37] C. Szegedy, W. Liu, Y. Jia, P. Sermanet, S. Reed, D. Anguelov, et al., "Going Deeper with Convolutions," Proc. of the IEEE Conference on Computer Vsion and Pattern Recognition, pp. 1-9, October 2015.

[38] J. W. Liu, F. L. Zuo, Y. X. Guo, T. Y. Li, and J. M. Chen, "Research on Improved Wavelet Convolutional Wavelet Neural Networks," Applied Intelligence, vol. 50, no. 11, pp. 1-21, November 2020.

Copyright $($ by the authors. Licensee TAETI, Taiwan. This article is an open access article distributed under the terms and conditions of the Creative Commons Attribution (CC BY-NC) license (https://creativecommons.org/licenses/by-nc/4.0/). 